\title{
GPS-Prot: A web-based visualization platform for integrating host-pathogen interaction data
}

Marie E Fahey ${ }^{1,2+}$, Melanie J Bennett ${ }^{2 \dagger}$, Cathal Mahon ${ }^{1,3}$, Stefanie Jäger ${ }^{1}$, Lars Pache ${ }^{4}$, Dhiraj Kumar ${ }^{5}$, Alex Shapiro ${ }^{6}$, Kanury Rao ${ }^{5}$, Sumit K Chanda ${ }^{4}$, Charles S Craik ${ }^{3}$, Alan D Frankel ${ }^{2^{*}}$ and Nevan J Krogan ${ }^{1^{*}}$

\begin{abstract}
Background: The increasing availability of HIV-host interaction datasets, including both physical and genetic interactions, has created a need for software tools to integrate and visualize the data. Because these host-pathogen interactions are extensive and interactions between human proteins are found within many different databases, it is difficult to generate integrated HIV-human interaction networks.

Results: We have developed a web-based platform, termed GPS-Prot http://www.gpsprot.org, that allows for facile integration of different HIV interaction data types as well as inclusion of interactions between human proteins derived from publicly-available databases, including MINT, BioGRID and HPRD. The software has the ability to group proteins into functional modules or protein complexes, generating more intuitive network representations and also allows for the uploading of user-generated data.

Conclusions: GPS-Prot is a software tool that allows users to easily create comprehensive and integrated HIV-host networks. A major advantage of this platform compared to other visualization tools is its web-based format, which requires no software installation or data downloads. GPS-Prot allows novice users to quickly generate networks that combine both genetic and protein-protein interactions between HIV and its human host into a single

representation. Ultimately, the platform is extendable to other host-pathogen systems.
\end{abstract}

\section{Background}

The application of high-throughput, unbiased, "systems" approaches to study host-pathogen relationships is facilitating a shift in focus from the pathogen to the response of the host during infection. A more global view of the physical, genetic and functional interactions that occur during infection will provide a deeper insight into the regulatory mechanisms involved in pathogenesis and may eventually lead to new cellular targets for therapeutic intervention.

Currently, the vast majority of host-pathogen physical interaction data involves HIV, for which a large amount of physical binding information has historically been available, mostly from small-scale, hypothesis-driven experiments [1]. For example, the HIV-1 Human

\footnotetext{
* Correspondence: frankel@cgl.ucsf.edu; krogan@cmp.ucsf.edu

+ Contributed equally

'Department of Cellular and Molecular Pharmacology, University of California San Francisco, 1700 4th Street, San Francisco, 94158 USA

${ }^{2}$ Department of Biochemistry and Biophysics, University of California San

Francisco, 600 16th Street, San Francisco, 94158 USA

Full list of author information is available at the end of the article
}

Protein Interaction Database (HHPID) maintained by NIAID contains over 2500 functional connections between individual and human proteins observed over 25 years of research, approximately $30 \%$ of which are classified as physical binding interactions [2]. Another database, VirusMINT [3], contains a collection of literature-curated physical interactions for several viruses, the vast majority corresponding to HIV-1.

Several large-scale, systematic studies using the yeast two-hybrid methodology have recently been performed for several important human pathogens, including hepatitis C [4], Epstein-Barr [5], and influenza [6] viruses. Other approaches, such as those using Protein-fragment Complementation Assays (PCA) [7], protein arrays [8], or affinity tagging/purification combined with mass spectrometry (AP-MS) [9], which have been successfully used in other systems [10-13], have not been exploited to systematically interrogate host-pathogen physical relationships. We have, however, recently carried out the first systematic host-pathogen AP-MS study targeting HIV-1 using two different cell lines (HEK293 and

\section{() Biomed Central}


Jurkat) (Jager et al., submitted), which will further increase the need for tools to visualize and integrate host-pathogen interaction datasets.

In addition to physical interaction studies, functionally important factors in HIV biology have also been identified by genetic or proteomic profiling screens. These studies do not necessarily identify physical binding partners for pathogenic proteins, but rather often implicate pathways or indirect "functional" associations. In 2008, three separate siRNA screens were published (Brass, Konig, and Zhou datasets) [14-16] that identified host genes required for efficient HIV infection. More recently, an additional RNAi screen was carried out using shRNAs in a potentially more physiologically relevant Jurkat cell line (Yeung dataset) [17]. RNAi studies in mammalian cells are also giving new insights into the host response to a number of other pathogenic organisms, including hepatitis C $[18,19]$, influenza [20-23], West Nile [24], and Dengue fever viruses [25].

Similarly, several mass spectrometry-based studies examined protein expression levels in HIV-infected and uninfected cells. For example, Speijer and colleagues [26] used a 2D-DIGE approach in the human T-cell line PM1 where protein expression was measured following HIV infection. Another study examined protein abundance changes in a CD4 cell line 36 hours post-infection [27], whereas the most recent study reports on global protein level changes in primary CD4 cells isolated from five donors [28], profiling proteomic changes post infection in a time-dependent fashion.

At the most basic level, there exist two different types of data (physical vs. functional) and they both provide different insights into molecular mechanism. For example, genetic and proteomic profiling screens probing HIV-human interactions provide a wealth of data on genes and processes that contribute to pathogenesis but do not necessarily reflect direct physical connections. Conversely, methodologies that probe for physical interactions often miss crucial functional connections. Therefore, poor overlap is often seen when comparing datasets derived from these different, but complementary platforms. However, even a comparison of datasets collected using the same technology can reveal a very low overlap. For example, although the initial HIV RNAi screens each identified approximately 300 genes [14-16], there was a small (albeit statistically significant) overlap of three factors $[29,30]$. Several reasons contribute to this lack of concordance, including differences in the cell types (e.g., HeLa vs. HEK293T), the RNAi approaches and libraries used, as well as the phenotypic effects that were monitored. A comparison of all four genetic screens, which includes the most recent dataset derived from Jurkat cells using an shRNA library [17], finds no common factor between them (Figure 1A). In fact, only seven of 252 genes in this dataset are shared with even one of the other genetic screens $(p=0.654)$. Similarly, proteomic profiling datasets shared a low number of proteins (three) among all three datasets, although this is still statistically significant $\left(\mathrm{p}<10^{-5}\right.$, Figure 1B).

In cases where multiple types of data are available, it has been extremely illuminating to combine the diverse datasets to identify common pathways, processes, and complexes. For example, one recent study combined genetic and physical interaction data to identify new regulators of $\mathrm{Wnt} / \beta$-Catenin signaling in mammalian cells [31]. Another study carried out a meta-analysis of several host-HIV-1 datasets, integrated with host protein-protein interaction databases, and reported significant overrepresented clusters within a network of host-
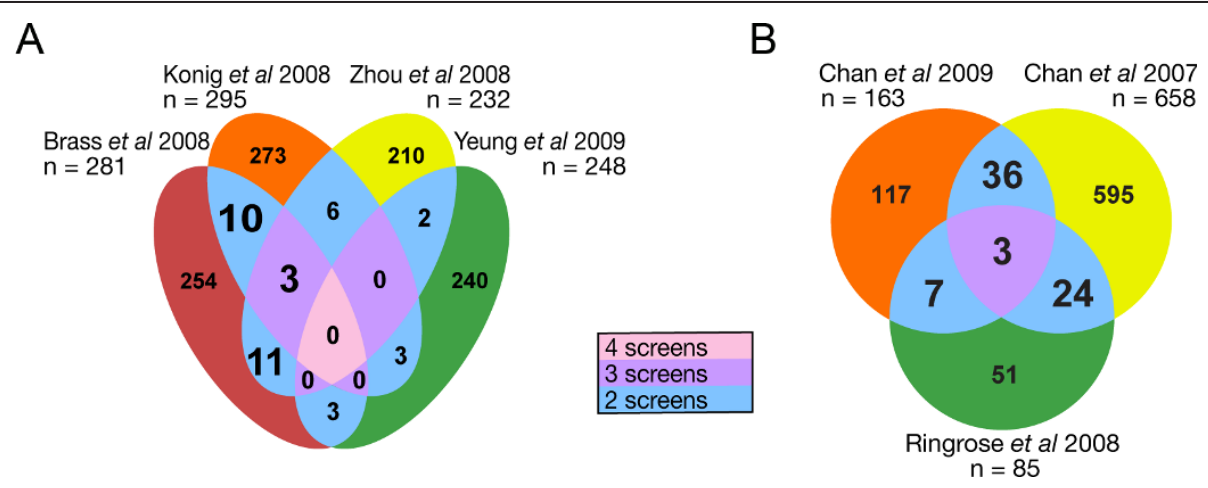

Figure 1 Numerous host factors have been identified for HIV by small-scale and high-throughput experiments, with little overlap between the various sources. (A) Venn diagram shows overlap from four HIV-based genetic screens [14-17]. Only three intersections show a significantly higher number of shared genes than expected, which are highlighted in large type. Ten genes are shared between the Brass and König datasets $(p=0.01)$, 11 between Brass and Zhou datasets $(p=0.0014)$, and three between Brass, König, and Zhou datasets $\left(p=5 \times 10^{-5}\right)$. None are shared between all four datasets. (B) Venn diagram shows a similar analysis for three HIV-dependent proteomic profiling screens [26-28]. Large type highlights statistically significant overlaps between the datasets (below $1 \times 10^{-4}$ ). 
pathogen and host-host interactions as important functional modules involved in virulence [29]. Another recent study identified key processes and host cellular subsystems impacted by HIV-1 infection by analyzing patterns of interactions in the HHPID, in combination with functional annotation and cross-referencing to global siRNA data [32].

In order to facilitate integration and exploration of the vast number of HIV-human interactions from different databases and data types, we have created a tool, termed GPS-Prot, with access to all major HIV-1 and human interaction databases as well as an option to overlay functional data (e.g. genetic interactions), which requires only very basic user input to produce an integrated network. To our knowledge this is the first tool to combine comprehensive HIV-1 and human physical/functional interaction data with a graphical viewer and web interface. Users can thus apply the GPS-Prot platform as a "global positioning system" to visualize any human-HIV1 interaction in the context of its landscape of reported binding partners. We have also implemented a feature for users to securely upload and view their own datasets of interest. This software uses a unique graphical interface based on TouchGraph LLC's Navigator program, which has been used for social networking applications and which makes navigating and gathering information from large networks intuitive and rapid. We therefore suggest that GPS-Prot is ideal for a novice user to quickly and easily build human-HIV-1 interaction networks from the wealth of published information, or from a user's own dataset, and to expand the network around a particular protein of interest.

\section{Implementation}

\section{Analysis of overlapping genes/proteins}

Gene lists were obtained from four genetic screens [14-17] and three proteomic profiling studies [26-28] and converted to NCBI Entrez gene identifiers. A list of published and converted identifiers for all screens can be found in Additional file 1 (see Additional file 1: identifiers.xls). Statistical significance of gene/protein overlaps was calculated using frequency of overlap in sizematched, randomly generated datasets.

\section{Development of GPS-Prot}

GPS-Prot is hosted on an Apache 2.0 web server and data retrieved from external databases resides in a MySQL relational database. Identifiers are mapped to Entrez GeneIDs. The logic tier is handled by PHP5 and the output of each database search is an XML file describing (1) individual proteins and (2) binary interactions. This file is passed to the network viewer, a version of TouchGraph Navigator (java applet) that is customized for our application. A spring-embedded layout is created within Navigator to view and navigate through the network, along with data tables containing information about the proteins and interactions. The Navigator applet performs well with up to 100,000 nodes and 200,000 edges, which is larger than any network that typical users will encounter. A connection to the server can be established within the applet allowing subsequent searches to be carried out by double-clicking on proteins in the network with the new interactions being added to the existing network.

Human PPIs are taken from six publicly available human interaction databases (downloaded June 2011; to be updated quarterly): HPRD [33] (Release 8), IntAct [34], MINT [35], BioGRID [36], DIP [37], and MIPS [38]. VirusMINT [3] (downloaded June 2011, to be updated quarterly) is used as the default HIV-human interaction database in GPS-Prot. Each interaction is linked to PubMed identifiers (PMID) and experimental descriptors and all protein identifiers are converted to Entrez gene nomenclature to facilitate identification of duplicate entries, which are consolidated for scoring purposes. The seven functional screens discussed here are also searched by default (1763 factors).

Additional optional databases currently include HIVBIND (a subset of BIND containing HIV-human interactions) [39], the NIAID HIV-1 Human Database (HHPID) [40] from which many of the interactions in VirusMINT are derived, CORUM [41], and a published set of predicted HIV-human interactions (3372 interactions) [42].

To simplify searching and viewing, we do not separate viral proteins according to strains. All interactions imported from the various databases are mapped to the representative virus protein name.

To facilitate visualization of large networks, each physical interaction in the network is assigned a score. A high score indicates that an interaction has been reported in several independent publications, or perhaps only once, but with a high-confidence experimental technique (e.g. NMR or x-ray crystallography). The method is a modification of that used by the MINT database [35], which has been adapted for use across multiple databases, where curation standards and reported details of experiments vary (see Additional file 2; Additional_methods.doc). The optional database of CORUM complexes is treated as if all subunits interact and scored as 1.0 so that they are retained in the networks at any scoring threshold. The output of a search is an XML file, viewed using a customized applet for PPIs that appears in the GPS-Prot Navigator window (TouchGraph LLC, New York, NY).

User upload of data (up to nine datasets) is permitted after creating an account at the GPS-Prot website. Uploaded data can be of two types: physical interactions 
or genetic/functional interactions. Physical interactions should be formatted as a two-column list of interacting proteins (Uniprot or Entrez identifiers, tab delimited; e. g., .txt file from Microsoft Excel). Genetic/functional interactions should be formatted as a single column list of Uniprot or Entrez identifiers. At present, only HIV or human proteins can be uploaded.

\section{Analysis of overlapping complexes/functional modules}

Datasets were analyzed in terms of subunits of complexes or functional modules defined by CORUM [41]. Because CORUM includes subunits interacting with multiple complexes or subcomplexes, we created an all-against-all binary matrix of protein interactions to assign subunits to unique complexes or functional modules. This was necessary to assign one complex and its subunits to one intersection of the datasets. Hierarchical clustering was carried out on the matrix using Cluster 3.0 and a branch length threshold of 1.6 was used to select clusters from the dendrogram, which we defined as our set of complexes, after some manual refinement (see Additional file 3: Corum_compl.xls). In total, the set consists of 222 complexes, containing 1600 subunits (see Additional file 3: Corum_compl.xls). Genes/proteins from the datasets were assigned to complexes/functional modules and the overlaps of complexes between the different datasets calculated. Statistical significance of the number of subunits overlapping was calculated using frequency observed in size-matched, randomly generated datasets. In addition, the significance of the number of subunits identified in each complex was calculated using the hypergeometric distribution function in Microsoft Excel, (see Additional files 4 and 5: RNAi_compl.xls and Prot_compl.xls).

\section{Identification and verification of Vif complexes}

Vif-binding proteins were identified by affinity tagging/ purification combined with mass spectrometry analysis (Jager et al., submitted). To investigate further the novel interaction with Huwe1, we performed immunoprecipitations and Western blotting as follows: Plasmids that express Vif, Vpr, or Nef were constructed by inserting cDNA-derived genes into a pcDNA3 vector containing C-terminal tandem 2xStrep/3xFLAG tags, and 293 cells were transfected using calcium phosphate. Cells were harvested two days post-transfection and lysed and immunoprecipitated with anti-FLAG M2 affinity resin (Sigma) according to manufacturer instructions. Proteins eluted with 3xFLAG peptide were analyzed by Western blot using anti-Cul5, anti-UPF1 and anti-Elongin B (TCEB2) (Santa Cruz), anti-FLAG (Sigma), or antiHuwe1 (Bethyl Laboratories) antibodies. Western blots were developed using ECL Plus Western Blotting Detection System (GE Healthcare).

\section{Results}

Generation of HIV-1-human networks using GPS-Prot

The GPS-Prot platform, found at http://www.gpsprot. org, allows users to initiate searches either by selecting an HIV protein from a graphic of the viral genome or by entering an HIV or human gene identifier in the search box (Figure 2A). A network is then generated and visualized (Figure 2B) using data from several publicly-available protein interaction databases, including VirusMINT [3] for HIV-host interactions, and HPRD [33], IntAct [34], MINT [43], BioGRID [36], DIP [37] and MIPS [38] for interactions between human proteins. There are also additional databases that can be selected.

The GPS-Prot databases selected on the homepage can also be searched from within the Navigator window by double clicking any node. Thus, it is possible to visualize not only the HIV-host interactions but also to explore second-shell (or third-shell, etc.) host-host interactions in an intuitive manner. Figure $2 \mathrm{~B}$ shows a network with all human binding partners to the HIV Vif protein. In this case, after the initial network of Vif binders was built, the binding partners of CUL5, a factor hijacked by Vif [44], were added into the network by double clicking the CUL5 node (Figure 2B, right-most network).

Two text panels are located to the left of the network window. The top panel toggles to display two types of information depending on what is selected in the network: details about any protein (node) or any interaction (edge) (e.g. panels headed "CUL5" and "Interactions", respectively) (Figure 2B). Single clicking any node or edge toggles between the windows and includes information about the originating database(s) for the PPI (protein-protein interaction), experiment type, links to publications, functional information, and Uniprot entries.

Two tabs in the bottom left panel allow users to toggle between two tables that provide further details about the network. The "Protein" tab lists all proteins or nodes while the "Interactions" tab lists all interactions or edges. By default, a limited amount of information is included for each protein or interaction, which can be expanded to include additional parameters. For example, a useful "keywords" field can be added to the interactions table when using the NIAID HHPID database, and then interactions can be sorted by clicking on the column headers. Groups of table entries can be selected (e. g. all having the same keyword), causing them to be highlighted in the network panel. The search box can be used to find any particular protein in the loaded network.

We have assigned rough "confidence scores" to each pair-wise interaction based on the number of 


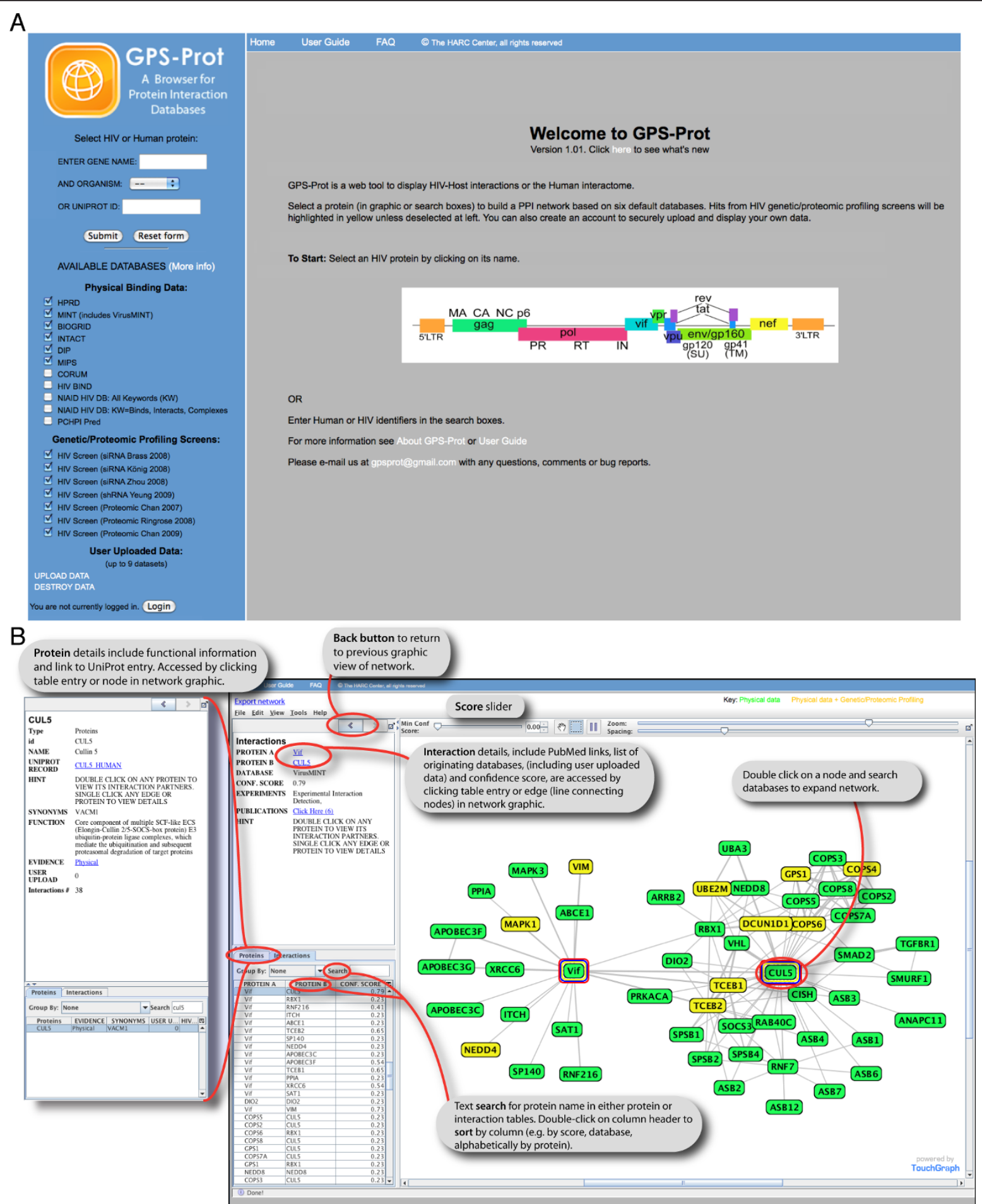

Figure 2 GPS-Prot: a web-based platform for visualizing diverse HIV-host data. (A) GPS-Prot homepage. Searches are initiated by selecting databases and an HIV or host protein. (B) A Touchgraph Navigator window is launched to display results of a search, which contains the protein interaction network. Single clicking any interaction ("edge", or gray line connecting proteins) provides the evidence from the literature for that interaction in the left-hand panel. Clicking on any protein in the diagram ("node") pulls up details for that protein (e.g. panel labeled CUL5). There is also a searchable table that can be sorted by score, database or experiment. A new network can be created by double clicking any protein (node), thus, it is possible to "walk through" the entire HIV-human or human-human interactome. 
independent publications and experimental methods (see Implementation), similar in concept to the scoring used by the MINT database [43]. However, the scores used by GPS-Prot are not meant to evaluate the validity of interactions in any absolute way, but rather to allow users to dynamically change the number of viewed nodes by adjusting a confidence score slider in the network panel (Figure 2B), thereby acting as a filter to help visualize large networks with many nodes. The edge line widths in the network panel are also displayed in proportion to their scores and future quantitative information about HIV-human interactions can be incorporated later. For example, we have devised the MiST (mass spectrometry interaction statistics) score to quantitatively report on interactions derived from systematic AP-MS studies (Jager et al., submitted) and these values can be effectively incorporated into GPS-Prot.

The Navigator window also includes other features to help simplify visualization, such as zoom and spacing sliders (Figure 2B) and the ability to resize the information and network panels by dragging borders. Network images can be exported using a "Save Image" option under the File pulldown menu. Data can also be exported in the form of a tab-delimited file by using the "Export network" link in the Navigator window.

\section{Overlay of physical and functional interaction networks}

One challenge in handling large-scale genomic datasets is the difficulty in integrating different data types, a task accomplished in GPS-Prot by allowing users to view data from functional screens in the context of PPI networks. By default, GPS-Prot includes seven genetic and proteomic profiling screens carried out in the context of HIV-1 infection [14-17,26-28], which are overlaid on the physical binding networks (Figure 2). Operationally, the physical interaction network is first built from the PPI databases (green nodes) and then interactors identified by the genetic or proteomic screens are highlighted in yellow, with links to publications in the information panel. Including functional data in a GPS-Prot search can highlight relevant clusters in a network. For example, the well-established complex of Vif with TCEB1 (Elongin C), TCEB2 (Elongin B) (which forms a larger complex with the Ring Box protein RBX1, and CUL5) [44], is easily noted in Figure 2B, as the Elongin subunits are highlighted in yellow based on RNAi and proteomic profiling screens. The importance of this complex during the HIV life cycle is well appreciated, as Vif targets APOBEC3G for degradation during the course of infection [44].

\section{Use of CORUM to identify complexes involved in HIV function}

Another important feature of GPS-Prot is the ability to group subunits of complexes together by including data from the CORUM database [41], a collection of manually curated mammalian protein complexes. To date, there are several examples of HIV proteins interacting with well-characterized human complexes. For example, Tat interacts with CCNT1/CDK9, components of the elongation factor $\mathrm{pTEFb}$, along with the chromatin regulators, AFF4, ENL, ELL, and AF9 [45,46], a complex important for transcriptional activation, and as previously mentioned, Vif hijacks a multi-subunit ubiquitin ligase complex containing Cul5, thus targeting APOBEC3G to the proteasome for degradation [44]. Analyzing and visualizing datasets in terms of complexes can increase agreement between different functional screens, which often have little overlap at the individual gene or protein level (Figure 1; [29]).

We used the CORUM database to identify statistically significant overlaps between genetic and proteomic screens. Initially, we found that the four HIV RNAi screens [14-17] are enriched for proteins that are part of protein complexes (Figure 3A), as annotated by CORUM. This trend was also observed for other small viruses for which RNAi data is available (Figure 3A), including hepatitis $C[18,19]$ and influenza $[20,22,23]$. To see how these trends compared to genetic data derived from a bacterial pathogen, we analyzed a recent RNAi screen that assessed effects of Mycobacterium tuberculosis (Mtb) infection [47]. In this case we found no strong enrichment for subunits of protein complexes within the dataset (Figure 3A, p = 0.05). This was not due to an abundance of weakly expressing genes in the Mtb screen that could cause under-representation in the CORUM database (Additional file 6; Figure S1.doc). The observation that HIV and other viruses appear to target larger molecular machines compared to Mtb is consistent with the hypothesis that its significantly smaller genome (15 proteins vs. 4000 in Mtb) requires that it needs to physically hijack a greater proportion of the host machinery.

Our analysis also shows that HIV-1 RNAi datasets have a greater intersection when they are analyzed in terms of multi-subunit complexes rather than as individual factors. The tables in Figure 4 show the number of subunits from the same complex identified in the RNAi (Figure 4A) and proteomic screens (Figure 4B). For example, both the spliceosome and proteasome were identified in all four genetic screens and included 34 subunits $\left(\mathrm{p}=4.0 \times 10^{-4}\right)$ of these two complexes $(20$ and 14 subunits, respectively) $\left(\mathrm{p}=2.9 \times 10^{-6}, \mathrm{p}=4.8 \times\right.$ $10^{-9}$ respectively) (Additional file 4:RNAi_compl.xls). In all, 48 proteins $\left(\mathrm{p}=1.7 \times 10^{-4}\right)$ belonging to eight separate complexes and 40 proteins $\left(\mathrm{p}=2.5 \times 10^{-3}\right)$ belonging to 17 separate complexes were identified in three and two screens, respectively (Additional file 4: RNAi_compl.xls). Collectively, there were 1014 proteins 

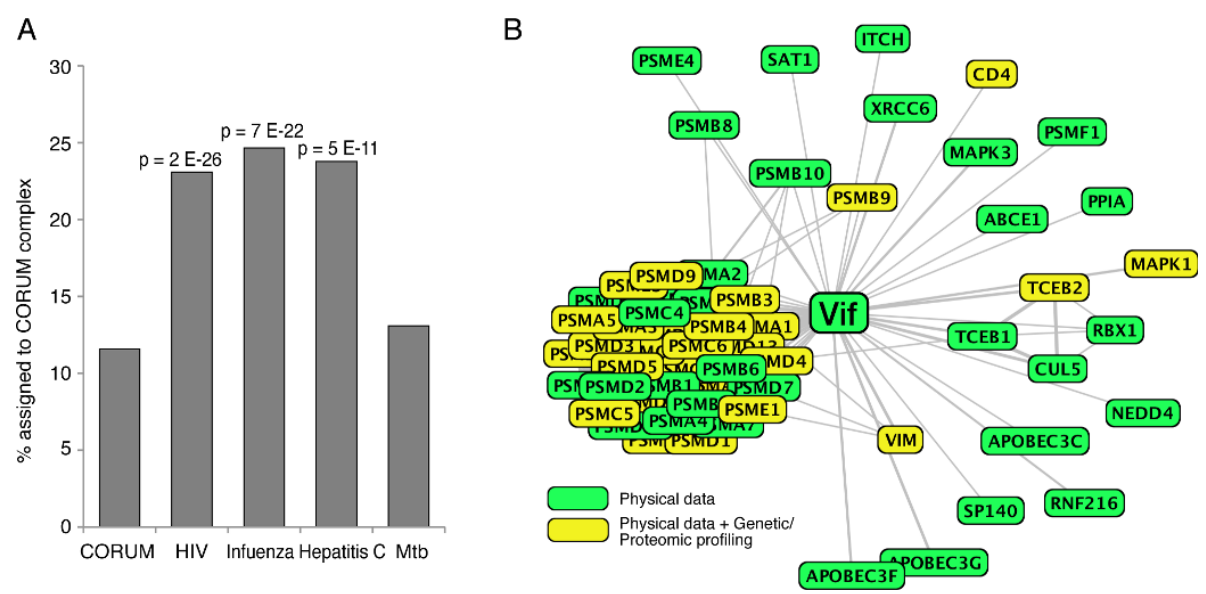

Figure 3 Viral RNAi screens are enriched for host factors that are subunits of human complexes. (A) All viral RNAi screens identify significantly more human complex subunits identified than expected (HIV 23\%, influenza 25\%, and hepatitis C 24\%), compared to the number of proteins in the human genome assigned to complexes by CORUM (12\%). P values shown are based on the hypergeometric distribution. We find no strong enrichment of protein complexes in a screen of Mtb host factors (13\%). (B) Network of Vif interactors from GPS-Prot using the optional NIAID HIV-1-human interactions database, instead of VirusMINT. Including CORUM as a database brings complex subunits closer together in the network, for example the cluster of proteasome complex subunits shown to the lower left (e.g. PSMA, PSMB, PSMC, etc).

identified in all four RNAi screens, of which 122 are found in at least two screens when analyzed in the context of a protein complex $\left(\mathrm{p}<10^{-5}\right)$.

A similar concordance is found in the proteomic profiling datasets when analyzed in the context of protein complexes (Figure 4B, Additional file 5:Prot_compl.xls). In total, 120 complexes are implicated in HIV function by all seven datasets (Additional files 4 and 5: RNAi_compl.xls and Prot_compl.xls). Some complexes were identified by both technologies, including the proteasome (Figure 4A and 4B), while others were only significantly enriched in one, such as ESCRT III in the proteomic profiling screens. Overall, 38 complexes are identified by both genetic and proteomic profiling, 48 by genetic screening alone, and 34 by proteomic profiling alone.

To confirm this analysis, we sought to verify one of these identified complexes experimentally. This was accomplished by knockdown of a set of mediator subunits that were not identified in any screen as host factors (gray subunits in Figure 4). We found that RNAi targeted to one of these, MED30, strongly inhibited early-stage HIV replication without inducing toxicity (Additional file 7; Figure S2.doc). MED30 is contained within the head module of Mediator, one of four functionally distinct sub-complexes [48], and is required for promoter recognition [49] and assembly/stabilization of transcription pre-initiation complexes [50,51]. Interestingly, RNAi knockdown of 8 out of $11(\mathrm{p}=0.007)$ head module factors (including MED30) affect replication while no protein in the Cdk8 module was identified in any of the RNAi screens (see Additional file 4: RNAi_compl.xls).
Based on this analysis, we conclude that analyzing the genetic data in the context of complexes is useful for identifying statistically significant factors affecting HIV function. Allowing users to optionally select CORUM in GPS-Prot permits a similar analysis, albeit at a visual level, by highlighting complexes with different subunits that have been identified in different screens. We have found that including data from the CORUM database can increase the visual overlap between different genetic and proteomic screens and allow users to disentangle biochemical complexes from broader biological processes. Figure 3B shows the visual advantage of including CORUM in a search; in this case, using it in conjunction with the NIAID HIV-1-human interactions database. GPS-Prot presumes an edge between all members of a complex, bringing members in the network into a very dense cluster of nodes. As shown in Figure 4 , different subunits of the proteasome are identified in all seven HIV functional screens. The proteasome is much more clearly identified as a complex, in GPS-Prot when CORUM data is included.

The approach of combining information from different screens, particularly those utilizing different technologies, is effective, in part, because many screens do not reach saturation. There can also be a high false negative rate (e.g. known binders of HIV proteins, such as Cyclin $\mathrm{T} 1$, are not found in some screens) or false positive rate, due to off target effects and variable expression of host factors in different cell lines. Analyses in the context of complexes compensates to some extent for these limitations by identifying overlaps between datasets, especially when saturation is not reached. 


\section{Upload of user-generated data}

According to the HHPID database, numerous host factors (up to several hundred) may interact with any given HIV-1 protein. In addition, RNAi screens alone have added more than 800 unique host factors to the current datasets. The continuing issue when obtaining new datasets is to distinguish between relevant hits and noise, which can be aided, as we have shown, by combining multiple datasets and/or analyzing the data in the context of protein complexes. To address this need, GPSProt allows users to create an account and upload up to nine in-house datasets to be included in the interaction networks. The set can describe physical interactions, consisting of a list of binary interacting proteins, or simply a list of genes/proteins such as that generated by RNAi or proteomic profiling screens (see Implementation for details).

We used this feature to analyze a partial dataset from our ongoing project to determine a comprehensive human-HIV-1 interaction map using AP-MS [52] (Jager et al., submitted). We obtained preliminary interaction data for Vif by transiently expressing and purifying a Cterminally 3xFLAG tagged version from HEK293 cells and analyzed the associated proteins by mass spectrometry. We then uploaded these data into GPS-Prot, to view in the context of previously reported Vif binders (Figure 5A; uploaded data are marked with red tags). The most well-characterized Vif partners, TCEB1 (Elongin C), TCEB2 (Elongin B), and CUL5 (circled in red and highlighted in the lower left table), were present in the AP-MS dataset and two of these (TCEB1 and TCEB2) were also found in RNAi and/or proteomic screens (yellow nodes). Interestingly, of the four remaining proteins observed both by AP-MS and in the screens (yellow and red-tagged), three of these, PSME3 (a proteasome subunit), HUWE1 (an E3 ligase), and UBL4A (a ubiquitin-like protein), have functions that may relate to the role of Vif in ubiquitin-tagging substrates for proteasomal degradation. Because Huwe1 acts during the late stages of HIV infection [14] when Vif is believed to function, we retested the Vif-Huwe1 interaction by immunoprecipitation (IP)-Western blotting using an antibody against Huwe1 and indeed observed strong and specific binding (Figure 5B). It will be of great interest to determine whether Vif itself is targeted for ubiquitination by Huwe1 or whether Huwe1 might be a second ubiquitin ligase recruited by Vif to tag APOBEC3G or other as-yet-unidentified targets for degradation.

\section{Comparison with other platforms}

There are a number of tools for visually exploring biological networks, such as PINA [53], STRING [54], Cytoscape [55], and others (reviewed in [56]). Some standalone databases are also integrated with viewers, such as the MINT database [57]. Others are linked to external viewers such as Osprey [58] for BioGRID database interactions or the Cytoscape plugin MiSink for DIP interactions [59]. Alternatively, sites like STRING and APID/APID2NET have plug-ins for Cytoscape [60] and integrate interactome data from multiple PPI databases.

Many of the existing network analysis platforms, however, do not include HIV-host interactions, or virus-host interactions in general, and also require varying degrees of expert knowledge to produce and navigate networks. Thus, there is a need to integrate and synthesize the abundant HIV-host physical and genetic interaction information (or more generally host-pathogen information) from public repositories. PIG [61] and VirusMINT [3] have taken steps in this direction by creating databases that contain a substantial number of physical HIV interactions, along with other physical virus-host interactions. CAPIH is a tool that provides a web interface for accessing physical host-HIV interactions [62] in the context of comparative genome analysis and provides information about the differences in sequences between interacting proteins of model organisms (chimpanzee, rhesus macaque, and mouse). Also, a web version of JNets [63] allows users to view a global network representation of the HHPID HIV-host interactions and explore that network using the underlying annotations, such as Gene Ontology (GO) annotation or HHPID keywords.

Aside from the issue of integrating physical and genetic virus-host data, it has been noted that some biological network tools utilize generic graph drawing tools that are not necessarily intuitive to most biologists [56]. We took an alternative approach of harnessing a commercial viewer (TouchGraph Navigator), which has been developed for non-scientific applications including social network analysis, and modifying it in collaboration with its designers for our scientific application.

GPS-Prot also allows users to include information about complexes through inclusion of data from the CORUM database. Our results suggest this approach may be particularly suited to viruses or other pathogens that rely extensively on multi-subunit host machinery, as indicated by our preliminary comparison with the bacterial pathogen Mtb. However the vast majority of data available are from viral pathogens and more studies of microbe pathogens are required to definitively tease apart the differences.

\section{Conclusions}

As high-throughput technologies identify more host factors that physically associate with viral factors, it is vital to integrate this information with other, diverse types of data, such as genetic and proteomic profiling, and to 

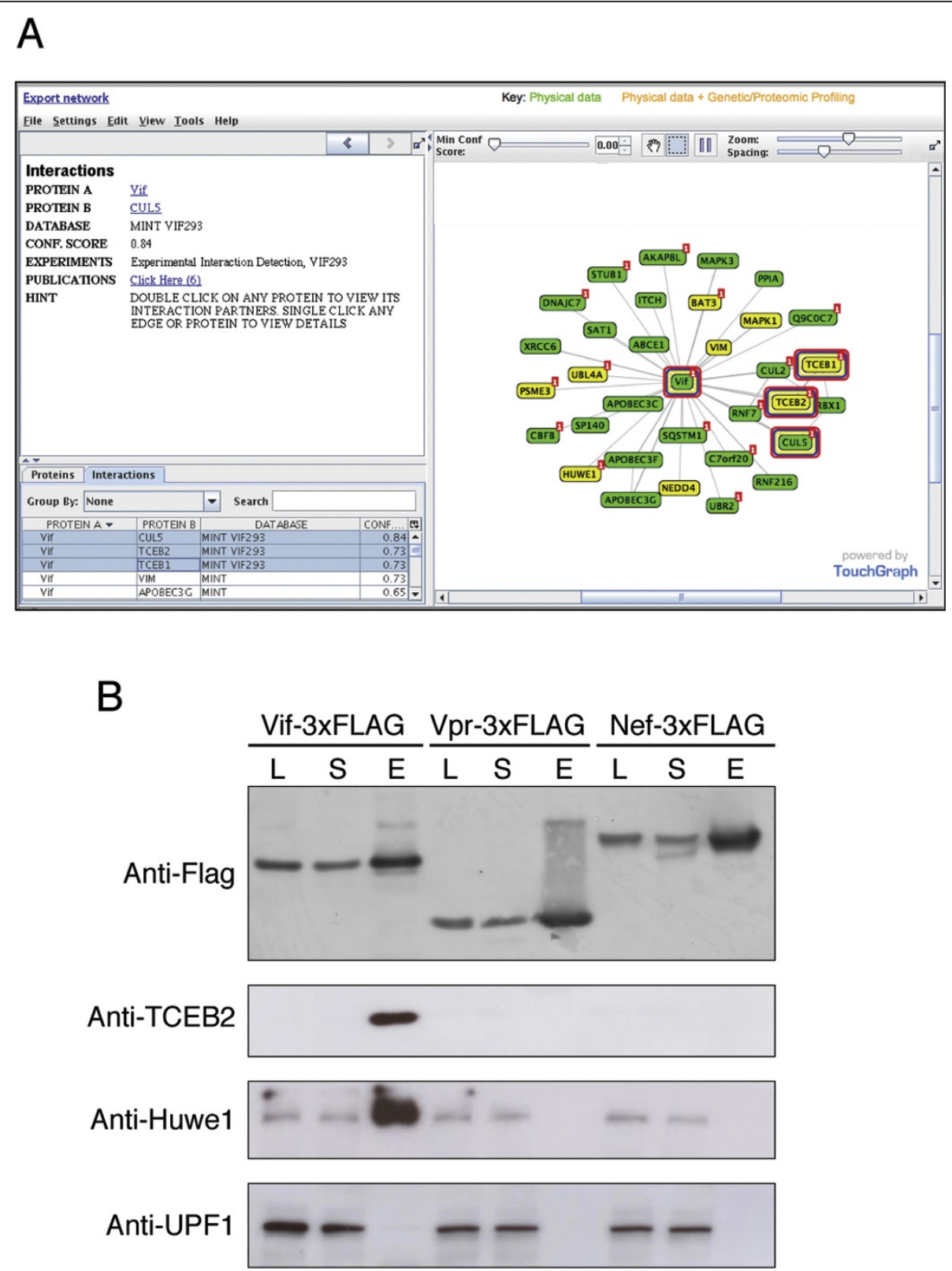

Figure 5 User-generated data can be uploaded and viewed in the context of complete PPI networks from public databases. (A) Vif network from GPS-Prot, including an uploaded dataset from AP-MS experiments (red-tagged nodes). Huwe1 is among several proteins in the uploaded dataset (Jager et al., submitted) that are not found in other databases (e.g., not present in Figure 2B), and were also previously identified by genetic/proteomic screens. (B) HIV Vif interacts with endogenous HUWE1 in 293 cells. 3xFLAG-tagged Vif, Vpr, and Nef were immunoprecipitated with anti-FLAG agarose beads. Lysates (L), remaining supernatant $(S)$ and eluates $(E)$ were analyzed by SDS-PAGE and Western blotting with antibodies as indicated. The same band is identified in the Vif pulldown by antibodies against the known CUL5 E3 ligase complex, anti-CUL5 (not shown) and anti-ELOB (TCEB2) as well as anti-Huwe1 antibodies, but not by the control anti-UPF1 antibody.

provide tools to visualize them in intuitive ways. GPSProt provides such a tool by aggregating several major databases for physical virus-host and host-host PPIs and overlaying HIV-1 genetic/proteomic profiling data, in addition to allowing upload of new user-generated data.

A next goal is to extend the GPS-Prot infrastructure to other pathogens, particularly viruses. Currently very few have datasets as large as HIV-1, particularly with regard to the physical interactome of each viral protein. We have collected physical interaction datasets derived from AP-MS studies for HIV-1 in HEK293 and Jurkat cells that will be included in the GPS-Prot set of databases (Jager et al., submitted). Finally, we also intend to expand these analyses to other pathogens in the near future. 


\section{Availability and Requirements}

GPS-Prot is freely available to all users with Javaenabled web browsers (best viewed with Safari and Firefox) at http://www.gpsprot.org. GPS-Prot was coded using XHTML, CSS, PHP, XML, Java, MySQL and jQuery.

\section{Additional material}

Additional file 1: Published and converted identifiers for all seven
HIV screens.
Additional file 2:
Additional file 3: Dataset of 222 human complexes derived from
CORUM by clustering and details of manual refinement of
complexes.
Additional file 4: Complexes and subunits identified by RNAi
studies.
Additional file 5: Complexes and subunits identified by proteomic
profiling studies.
Additional file 6: Comparison of broad expression level of Mtb and
HIV screens.
Additional file 7: RNAi-mediated depletion of MED30 blocks early
steps of replication of a VSV-G pseudotyped HIV luciferase virus.

List of Abbreviations

PPI: Protein-protein interaction

\section{Acknowledgements and Funding}

Vif DNA was obtained through the NIH AIDS Research and Reference Reagent Program, Division of AIDS, NIAID, NIH from Dr. Stephan Bour and Dr. Klaus Strebel and Vpr DNA was a kind gift from Michael Lenardo, $\mathrm{NIH}$. We thank Paul De Jesus for advice and excellent technical assistance with RNAi-based assays and Mike Shales for assistance with figure preparation. We are grateful to the UCSF Mass Spectrometry Facility (NIH grant P41RR001614), directed by Al Burlingame. This work was supported by NIH grants P50GM82250 to N. J. K., C.S.C. and A.D.F. and P01Al090935 to N. J. K. and S. K. C. N.J.K. is a Keck Young Investigator and Searle Scholar.

\section{Author details \\ ${ }^{1}$ Department of Cellular and Molecular Pharmacology, University of California San Francisco, 1700 4th Street, San Francisco, 94158 USA. ${ }^{2}$ Department of Biochemistry and Biophysics, University of California San Francisco, 600 16th Street, San Francisco, 94158 USA. ${ }^{3}$ Department of Pharmaceutical Chemistry, University of California San Francisco, 600 16th Street, San Francisco, 94158 USA. ${ }^{4}$ Sanford-Burnham Medical Research Institute, 10901 North Torrey Pines Road, La Jolla, 92037 USA. ${ }^{5}$ Immunology Group, International Centre for Genetic Engineering and Biotechnology, Aruna Asaf Marg, New Delhi 110 067, India. ${ }^{6}$ TouchGraph LLC, 306 W. 92nd Street \#3F, New York, 10025 USA.}

\section{Authors' contributions}

MEF, MJB, ADF and NJK designed the approach, analyzed data and wrote the manuscript. MEF, CM, SJ, LP, DK, KR, SKC, CSC collected results and analyzed data. All authors read and approved the final manuscript. MEF and MJB designed and implemented GPS-Prot website. AS designed and implemented customized Navigator applet.

\section{Competing interests}

The authors declare that they have no competing interests.

Received: 28 February 2011 Accepted: 22 July 2011

Published: 22 July 2011
References

1. Dyer MD, Murali TM, Sobral BW: The landscape of human proteins interacting with viruses and other pathogens. PLoS Pathog 2008, 4(2):e32.

2. Fu W, Sanders-Beer BE, Katz KS, Maglott DR, Pruitt KD, Ptak RG: Human immunodeficiency virus type 1, human protein interaction database at NCBI. Nucleic Acids Res 2009, 37 Database: D417-422.

3. Chatr-aryamontri A, Ceol A, Peluso D, Nardozza A, Panni S, Sacco F, Tinti M, Smolyar A, Castagnoli L, Vidal M, Cusick ME, Cesareni G: VirusMINT: a viral protein interaction database. Nucleic Acids Res 2009, , 37 Database: D669-673.

4. de Chassey B, Navratil V, Tafforeau L, Hiet MS, Aublin-Gex A, Agaugué S, Meiffren G, Pradezynski F, Faria BF, Chantier T, Le Breton M, Pellet J, Davoust N, Mangeot PE, Chaboud A, Penin F, Jacob Y, Vidalain PO, Vidal M, André $P$, Rabourdin-Combe C, Lotteau V: Hepatitis $C$ virus infection protein network. Mol Syst Biol 2008, 4:230.

5. Calderwood MA, Venkatesan K, Xing L, Chase MR, Vazquez A, Holthaus AM, Ewence AE, Li N, Hirozane-Kishikawa T, Hill DE, Vidal M, Kieff E, Johannsen E: Epstein-Barr virus and virus human protein interaction maps. Proc Natl Acad Sci USA 2007, 104(18):7606-7611.

6. Shapira SD, Gat-Viks I, Shum BOV, Dricot A, de Grace MM, Wu L, Gupta PB, Hao T, Silver SJ, Root DE, Hill DE, Regev A, Hacohen N: A physical and regulatory map of host-influenza interactions reveals pathways in $\mathrm{H} 1 \mathrm{~N} 1$ infection. Cell 2009, 139(7):1255-1267.

7. Tarassov K, Messier V, Landry CR, Radinovic S, Serna Molina MM, Shames I, Malitskaya Y, Vogel J, Bussey H, Michnick SW: An in vivo map of the yeast protein interactome. Science 2008, 320(5882):1465-1470.

8. MacBeath G, Schreiber SL: Printing Proteins as Microarrays for HighThroughput Function Determination. Science 2000, 289(5485):1760.

9. Puig O, Caspary F, Rigaut G, Rutz B, Bouveret E, Bragado-Nilsson E, Wilm M, Séraphin $B$ : The tandem affinity purification (TAP) method: a general procedure of protein complex purification. Methods 2001, 24(3):218-229.

10. Gavin A-CC, Aloy P, Grandi P, Krause R, Boesche M, Marzioch M, Rau C, Jensen LJ, Bastuck S, Dümpelfeld B, Edelmann A, Heurtier M-AA, Hoffman V, Hoefert C, Klein K, Hudak M, Michon A-MM, Schelder M, Schirle M, Remor M, Rudi T, Hooper S, Bauer A, Bouwmeester T, Casari G, Drewes G, Neubauer G, Rick JM, Kuster B, Bork P, et al: Proteome survey reveals modularity of the yeast cell machinery. Nature 2006, 440(7084):631-636.

11. Krogan NJ, Cagney G, Yu H, Zhong G, Guo X, Ignatchenko A, Li J, Pu S, Datta N, Tikuisis AP, Punna T, Peregrín-Alvarez JM, Shales M, Zhang X, Davey M, Robinson MD, Paccanaro A, Bray JE, Sheung A, Beattie B, Richards DP, Canadien V, Lalev A, Mena F, Wong P, Starostine A, Canete MM, Vlasblom J, Wu S, Orsi C, et al: Global landscape of protein complexes in the yeast Saccharomyces cerevisiae. Nature 2006, 440(7084):637-643.

12. Sowa ME, Bennett EJ, Gygi SP, Harper JW: Defining the human deubiquitinating enzyme interaction landscape. Cell 2009, 138(2):389-403.

13. Behrends C, Sowa ME, Gygi SP, Harper JW: Network organization of the human autophagy system. Nature 2010, 466(7302):68-76.

14. Brass AL, Dykxhoorn DM, Benita Y, Yan N, Engelman A, Xavier RJ, Lieberman J, Elledge SJ: Identification of host proteins required for HIV infection through a functional genomic screen. Science 2008, 319(5865):921-926.

15. König R, Zhou Y, Elleder D, Diamond TL, Bonamy GMC, Irelan JT, Chiang CYY, Tu BP, De Jesus PD, Lilley CE, Seidel S, Opaluch AM, Caldwell JS, Weitzman MD, Kuhen KL, Bandyopadhyay S, Ideker T, Orth AP, Miraglia LJ, Bushman FD, Young JA, Chanda SK: Global analysis of host-pathogen interactions that regulate early-stage HIV-1 replication. Cell 2008, 135(1):49-60.

16. Zhou H, Xu M, Huang Q, Gates AT, Zhang XD, Castle JC, Stec E, Ferrer M, Strulovici B, Hazuda DJ, Espeseth AS: Genome-scale RNAi screen for host factors required for HIV replication. Cell Host Microbe 2008, 4(5):495-504.

17. Yeung ML, Houzet L, Yedavalli VSRK, Jeang K-TT: A genome-wide short hairpin RNA screening of jurkat T-cells for human proteins contributing to productive HIV-1 replication. J Biol Chem 2009, 284(29):19463-19473.

18. Li Q, Brass AL, Ng A, Hu Z, Xavier RJ, Liang TJ, Elledge SJ: A genome-wide genetic screen for host factors required for hepatitis $C$ virus propagation. Proc Natl Acad Sci USA 2009, 106(38):16410-16415.

19. Tai AW, Benita Y, Peng LF, Kim S-SS, Sakamoto N, Xavier RJ, Chung RT: A functional genomic screen identifies cellular cofactors of hepatitis $C$ virus replication. Cell Host Microbe 2009, 5(3):298-307. 
20. Brass AL, Huang I-CC, Benita Y, John SP, Krishnan MN, Feeley EM, Ryan BJ, Weyer JL, van der Weyden L, Fikrig E, Adams DJ, Xavier RJ, Farzan M, Elledge SJ: The IFITM proteins mediate cellular resistance to influenza A H1N1 virus, West Nile virus, and dengue virus. Cell 2009, 139(7):1243-1254.

21. Hao L, Sakurai A, Watanabe T, Sorensen E, Nidom CA, Newton MA, Ahlquist P, Kawaoka Y: Drosophila RNAi screen identifies host genes important for influenza virus replication. Nature 2008, 454(7206):890-893.

22. Karlas A, Machuy N, Shin Y, Pleissner K-PP, Artarini A, Heuer D, Becker D, Khalil H, Ogilvie LA, Hess S, Mäurer AP, Müller E, Wolff T, Rudel T, Meyer TF: Genome-wide RNAi screen identifies human host factors crucial for influenza virus replication. Nature 2010, 463(7282):818-822.

23. König R, Stertz $S$, Zhou $Y$, Inoue A, Hoffmann H-HH, Bhattacharyya S, Alamares JG, Tscherne DM, Ortigoza MB, Liang Y, Gao Q, Andrews SE, Bandyopadhyay S, De Jesus P, Tu BP, Pache L, Shih C, Orth A, Bonamy G, Miraglia L, Ideker T, García-Sastre A, Young JAT, Palese P, Shaw ML, Chanda SK: Human host factors required for influenza virus replication. Nature 2010, 463(7282):813-817.

24. Krishnan MN, Ng A, Sukumaran B, Gilfoy FD, Uchil PD, Sultana H, Brass AL, Adametz R, Tsui M, Qian F, Montgomery RR, Lev S, Mason PW, Koski RA, Elledge SJ, Xavier RJ, Agaisse H, Fikrig E: RNA interference screen for human genes associated with West Nile virus infection. Nature 2008, 455(7210):242-245.

25. Sessions OM, Barrows NJ, Souza-Neto JA, Robinson TJ, Hershey CL, Rodgers MA, Ramirez JL, Dimopoulos G, Yang PL, Pearson JL, GarciaBlanco MA: Discovery of insect and human dengue virus host factors. Nature 2009, 458(7241):1047-1050.

26. Ringrose $\mathrm{JH}$, Jeeninga RE, Berkhout $B$, Speijer D: Proteomic studies reveal coordinated changes in T-cell expression patterns upon infection with human immunodeficiency virus type 1. J Virol 2008, 82(9):4320-4330

27. Chan EY, Qian W-JJ, Diamond DL, Liu T, Gritsenko MA, Monroe ME, Camp DG, Smith RD, Katze MG: Quantitative analysis of human immunodeficiency virus type 1-infected CD4+ cell proteome: dysregulated cell cycle progression and nuclear transport coincide with robust virus production. J Virol 2007, 81(14):7571-7583.

28. Chan EY, Sutton JN, Jacobs JM, Bondarenko A, Smith RD, Katze MG Dynamic host energetics and cytoskeletal proteomes in human immunodeficiency virus type 1-infected human primary CD4 cells: analysis by multiplexed label-free mass spectrometry. J Virol 2009, 83(18):9283-9295.

29. Bushman FD, Malani N, Fernandes J, D'Orso I, Cagney G, Diamond TL, Zhou H, Hazuda DJ, Espeseth AS, Konig R, Bandyopadhyay S, Ideker T, Goff SP, Krogan NJ, Frankel AD, Young JA, Chanda SK: Host cell factors in HIV replication: meta-analysis of genome-wide studies. PLOS Pathog 2009, 5(5):e1000437.

30. Goff SP: Knockdown screens to knockout HIV-1. Cell 2008, 135(3):417-420.

31. Major MB, Roberts BS, Berndt JD, Marine S, Anastas J, Chung N, Ferrer M, Yi X, Stoick-Cooper CL, von Haller PD, Kategaya L, Chien A, Angers S, MacCoss M, Cleary MA, Arthur WT, Moon RT: New regulators of Wnt/betacatenin signaling revealed by integrative molecular screening. Sci Signal 2008, 1(45):ra12.

32. Macpherson J, Pinney JW, Robertson DL: Patterns of HIV-1 protein interaction identify perturbed host-cellular subsystems. PLoS Comput Biol 2010, 6(7):e1000863.

33. Keshava Prasad TS, Goel R, Kandasamy K, Keerthikumar S, Kumar S, Mathivanan S, Telikicherla D, Raju R, Shafreen B, Venugopal A, Balakrishnan L, Marimuthu A, Banerjee S, Somanathan DS, Sebastian A, Rani S, Ray S, Harrys Kishore CJ, Kanth S, Ahmed M, Kashyap MK, Mohmood R, Ramachandra YL, Krishna V, Rahiman BA, Mohan S, Ranganathan P, Ramabadran S, Chaerkady R, Pandey A: Human Protein Reference Database-2009 update. Nucleic Acids Res 2009, , 37 Database: D767-772.

34. Kerrien S, Alam-Faruque $Y$, Aranda B, Bancarz I, Bridge A, Derow C, Dimmer E, Feuermann M, Friedrichsen A, Huntley R, Kohler C, Khadake J, Leroy C, Liban A, Lieftink C, Montecchi-Palazzi L, Orchard S, Risse J, Robbe K, Roechert B, Thorneycroft D, Zhang Y, Apweiler R, Hermjakob H: IntAct-open source resource for molecular interaction data. Nucleic Acids Res 2007, , 35 Database: D561-565.

35. Chatr-aryamontri A, Ceol A, Palazzi LM, Nardelli G, Schneider MV, Castagnoli L, Cesareni G: MINT: the Molecular INTeraction database. Nucleic Acids Res 2007, , 35 Database: D572-574.
36. Breitkreutz B-JJ, Stark C, Reguly T, Boucher L, Breitkreutz A, Livstone M, Oughtred R, Lackner DH, Bähler J, Wood V, Dolinski K, Tyers M: The BioGRID Interaction Database: 2008 update. Nucleic Acids Res 2008, , 36 Database: D637-640

37. Xenarios I, Salwínski L, Duan XJ, Higney P, Kim S-MM, Eisenberg D: DIP, the Database of Interacting Proteins: a research tool for studying cellular networks of protein interactions. Nucleic Acids Res 2002, 30(1):303-305.

38. Mewes HW, Frishman D, Mayer KFX, Münsterkötter M, Noubibou O, Pagel P, Rattei T, Oesterheld M, Ruepp A, Stümpflen V: MIPS: analysis and annotation of proteins from whole genomes in 2005. Nucleic Acids Res 2006, , 34 Database: D169-172.

39. Alfarano C, Andrade CE, Anthony K, Bahroos N, Bajec M, Bantoft K, Betel D, Bobechko B, Boutilier K, Burgess E, Buzadzija K, Cavero R, D'Abreo C, Donaldson I, Dorairajoo D, Dumontier MJ, Dumontier MR, Earles V, Farrall R, Feldman H, Garderman E, Gong Y, Gonzaga R, Grytsan V, Gryz E, Gu V, Haldorsen E, Halupa A, Haw R, Hrvojic A, et al: The Biomolecular Interaction Network Database and related tools 2005 update. Nucleic Acids Res 2005, , 33 Database: D418-424.

40. Ptak RG, Fu W, Sanders-Beer BE, Dickerson JE, Pinney JW, Robertson DL, Rozanov MN, Katz KS, Maglott DR, Pruitt KD, Dieffenbach CW: Cataloguing the HIV type 1 human protein interaction network. AIDS Res Hum Retroviruses 2008, 24(12):1497-1502.

41. Ruepp A, Brauner B, Dunger-Kaltenbach I, Frishman G, Montrone C, Stransky M, Waegele B, Schmidt T, Doudieu ON, Stümpflen V, Mewes HW: CORUM: the comprehensive resource of mammalian protein complexes. Nucleic Acids Res 2008, , 36 Database: D646-650.

42. Tastan O, Qi Y, Carbonell JG, Klein-Seetharaman J: Prediction of interactions between HIV-1 and human proteins by information integration. Pac Symp Biocomput 2009, 516-527.

43. Chatr-Aryamontri A, Zanzoni A, Ceol A, Cesareni G: Searching the protein interaction space through the MINT database. Methods Mol Biol 2008, 484:305-317.

44. Yu X, YU Y, Liu B, Luo K, Kong W, Mao P, Yu X-FF: Induction of APOBEC3G ubiquitination and degradation by an HIV-1 Vif-Cul5-SCF complex. Science 2003, 302(5647):1056-1060.

45. He N, Liu M, Hsu J, Xue Y, Chou S, Burlingame A, Krogan NJ, Alber T, Zhou Q: HIV-1 Tat and host AFF4 recruit two transcription elongation factors into a bifunctional complex for coordinated activation of HIV-1 transcription. Mol Cell 2010, 38(3):428-438.

46. Sobhian B, Laguette $N$, Yatim A, Nakamura M, Levy $Y$, Kiernan $R$, Benkirane M: HIV-1 Tat assembles a multifunctional transcription elongation complex and stably associates with the 7SK snRNP. Mol Cell 2010, 38(3):439-451.

47. Kumar D, Nath L, Kamal MA, Varshney A, Jain A, Singh S, Rao KVS: Genomewide analysis of the host intracellular network that regulates survival of Mycobacterium tuberculosis. Cell 2010, 140(5):731-743.

48. Paoletti AC, Parmely TJ, Tomomori-Sato C, Sato S, Zhu D, Conaway RC, Conaway JW, Florens L, Washburn MP: Quantitative proteomic analysis of distinct mammalian Mediator complexes using normalized spectral abundance factors. Proc Natl Acad Sci USA 2006, 103(50):18928-18933.

49. Takagi $Y$, Calero G, Komori H, Brown JA, Ehrensberger AH, Hudmon A, Asturias F, Kornberg RD: Head module control of mediator interactions. Mol Cell 2006, 23(3):355-364.

50. Cai G, Imasaki T, Takagi Y, Asturias FJ: Mediator structural conservation and implications for the regulation mechanism. Structure 2009, 17(4):559-567.

51. Cai G, Imasaki T, Yamada K, Cardelli F, Takagi Y, Asturias FJ: Mediator head module structure and functional interactions. Nat Struct Mol Biol 2010, 17(3):273-279

52. Jager S, Gulbahce N, Cimermancic P, Kane J, He N, Chou S, D'Orso I, Fernandes J, Jang G, Frankel AD, Alber T, Zhou Q, Krogan NJ: Purification and characterization of HIV-human protein complexes. Methods 2011, 53:13-19.

53. Wu J, Vallenius T, Ovaska K, Westermarck J, Mäkelä TP, Hautaniemi S: Integrated network analysis platform for protein-protein interactions. Nat Methods 2009, 6(1):75-77.

54. Szklarczyk D, Franceschini A, Kuhn M, Simonovic M, Roth A, Minguez $P$, Doerks T, Stark M, Muller J, Bork P, Jensen LJ, Mering CV: The STRING database in 2011: functional interaction networks of proteins, globally integrated and scored. Nucleic Acids Res 2011, 39:D561-D568. 
55. Shannon P, Markiel A, Ozier O, Baliga NS, Wang JT, Ramage D, Amin N, Schwikowski B, Ideker T: Cytoscape: a software environment for integrated models of biomolecular interaction networks. Genome Res 2003, 13(11):2498-2504.

56. Suderman M, Hallett M: Tools for visually exploring biological networks. Bioinformatics 2007, 23(20):2651-2659.

57. Ceol A, Chatr Aryamontri A, Licata L, Peluso D, Briganti L, Perfetto L, Castagnoli L, Cesareni G: MINT, the molecular interaction database: 2009 update. Nucleic Acids Res, 38 Database: D532-539.

58. Breitkreutz BJ, Stark C, Tyers M: Osprey: a network visualization system. Genome Biol 2003, 4(3):R22.

59. Salwinski L, Eisenberg D: The MiSink Plugin: Cytoscape as a graphical interface to the Database of Interacting Proteins. Bioinformatics 2007, 23(16):2193-2195

60. Hernandez-Toro J, Prieto C, De las Rivas J: APID2NET: unified interactome graphic analyzer. Bioinformatics 2007, 23(18):2495-2497.

61. Driscoll T, Dyer MD, Murali TM, Sobral BW: PIG-the pathogen interaction gateway. Nucleic Acids Res 2009, 37 Database: D647-650.

62. Lin F-KK, Pan C-LL, Yang J-MM, Chuang T-JJ, Chen F-CC: CAPIH: a Web interface for comparative analyses and visualization of host-HIV proteinprotein interactions. BMC Microbiol 2009, 9:164.

63. Macpherson JI, Pinney JW, Robertson DL: JNets: exploring networks by integrating annotation. BMC Bioinformatics 2009, 10:95.

doi:10.1186/1471-2105-12-298

Cite this article as: Fahey et al.: GPS-Prot: A web-based visualization platform for integrating host-pathogen interaction data. BMC

Bioinformatics 2011 12:298.

\section{Submit your next manuscript to BioMed Central} and take full advantage of:

- Convenient online submission

- Thorough peer review

- No space constraints or color figure charges

- Immediate publication on acceptance

- Inclusion in PubMed, CAS, Scopus and Google Scholar

- Research which is freely available for redistribution

Submit your manuscript at www.biomedcentral.com/submit
C Biomed Central 